\title{
Consumption of hot beverages and foods and the risk of esophageal cancer: a meta-analysis of observational studies
}

\author{
Yawen Chen ${ }^{1}$, Yeqing Tong ${ }^{2}$, Chen Yang ${ }^{1}$, Yong Gan ${ }^{1}$, Huilian Sun ${ }^{1}$, Huashan Bi ${ }^{1}$, Shiyi Cao ${ }^{1}$, Xiaoxv Yin ${ }^{\text {* }}$
} and Zuxun $\mathrm{Lu}^{1 *}$

\begin{abstract}
Background: Previous studies have mostly focused on the effects of specific constituents of beverages and foods on the risk of esophageal cancer (EC). An increasing number of studies are now emerging examining the health consequences of the high temperature of beverages and foods. We conducted a meta-analysis to summarize the evidence and clarify the association between hot beverages and foods consumption and EC risk.
\end{abstract}

Methods: We searched the PubMed, Embase, and Web of Science databases for relevant studies, published before May 1, 2014, with the aim to estimate the association between hot beverage and food consumption and EC risk. A random-effect model was used to pool the results from the included studies. Publication bias was assessed by using the Begg test, the Egger test, and funnel plot.

Results: Thirty-nine studies satisfied the inclusion criteria, giving a total of 42,475 non-overlapping participants and 13,811 EC cases. Hot beverage and food consumption was significantly associated with EC risk, with an odds ratio (OR) of 1.82 (95\% confidence interval [CI], 1.53-2.17). The risk was higher for esophageal squamous cell carcinoma, with a pooled OR of 1.60 (95\% Cl, 1.29-2.00), and was insignificant for esophageal adenocarcinoma (OR: 0.79; 95\% Cl: 0.53-1.16). Subgroup analyses suggests that the association between hot beverage and food consumption and EC risk were significant in Asian population (OR: 2.06; 95\% Cl: 1.62-2.61) and South American population (OR: 1.52; 95\% Cl: 1.25-1.85), but not significant in European population (OR: 0.95; 95\% Cl: 0.68-1.34).

Conclusions: Hot beverage and food consumption is associated with a significantly increased risk of EC, especially in Asian and South American populations, indicating the importance in changing people's dietary habits to prevent EC.

Keywords: Hot, Beverage, Food, Esophageal cancer, Meta-analysis

\section{Background}

Esophageal cancer (EC) is the eighth most common cancer in the world and ranks six among all cancers in mortality [1]. Many studies have shown that dietary habits are significantly correlated with the occurrence of EC $[2,3]$, most of which linking specific constituents of beverages and foods to EC. For example, Polyphenols in green tea was found to inhibit esophageal tumorigenesis [4], whereas maté infusion and caffeine appeared to induce mutagenic effects [5]. An increasing number of

\footnotetext{
*Correspondence: hnyinxiaoxv@126.com; zuxunlu@yahoo.com

${ }^{1}$ School of Public Health, Tongji Medical College, Huazhong University of

Science and Technology, Wuhan, Hubei, China

Full list of author information is available at the end of the article
}

studies have investigated the possible relationship between the temperature of beverages and foods and EC risk [6-8], since recurrent thermal injuries to the esophageal mucosa owing to the consumption of hot drinks or foods has long been considered a risk factor for EC [9].

Hot beverage consumption could substantially increase the intraesophageal temperature, depending on the initial drinking temperature. An animal study showed that the structure and the function of the esophageal epithelium were damaged by heat stress even [10]. However, epidemiological evidence on the causal relationship between the temperature of beverages and foods and EC is not well established. Research on the relationship was often done as a component of larger studies that focused 
on specific beverage or food gradients, and the results varied greatly across studies. Some studies found no association between hot beverages and foods and EC risk [11-13], arguing that the oral cavity could modulate the heat, and the temperature could fall too rapidly to cause injury to the esophageal mucosa [14]. But many other studies reported that the intake of hot beverages and foods increased EC risk [11,15,16].

In 2009, Islami and colleagues [9] reviewed fifty nine studies and found that over half of the studies showed statistically significant increased risk of EC associated with higher temperature of beverage and food intake. However, the authors did not use quantitative techniques to compute summary estimates of the risk, and the review is outdated. Therefore, we conducted this meta-analysis to ascertain the association between hot beverage and food consumption and EC risk more precisely, relying on all available evidence up-to-date, and to identify the potential factors affecting this association.

\section{Methods}

\section{Search strategy}

This meta-analysis was conducted according to the checklist of the Meta-Analysis of Observational Studies in Epidemiology Guideline [17]. We searched PubMed, Embase, and Web of Science databases from inception to May 1, 2014 for all epidemiological studies on hot beverage and food consumption in relation to EC risk, using the string '(esophageal OR oesophageal) AND (cancer OR carcinoma OR neoplasm) AND (tea OR maté OR coffee OR beverage OR liquid OR alcohol OR food OR diet)'. In addition, we scrutinized the reference lists from retrieved articles to identify other relevant studies.

\section{Inclusion criteria}

Studies were considered eligible for inclusion if they met the following criteria: (1) the study was a case-control or cohort study design, (2) it was published in English, (3) the exposure was hot beverage or food consumption, (4) the outcome of interest was EC, and (5) the study reported the odds ratio (OR) or relative risk (RR) with $95 \%$ confidence intervals (CIs) for the association between hot beverages or foods and EC risk or provided sufficient data to calculate them.

\section{Date extraction}

We extracted the following data from each retrieved article: name of first author, publication year and country of study, study design, specific outcomes, characteristics of study population, number of cases and participants, exposure type, exposure measurement, outcome assessment, comparison categories, OR or RR and corresponding $95 \% \mathrm{CI}$, and confounding factors adjusted in the analyses. Data from included studies were independently extracted by two authors (Y.W.C and Y.C), and disagreements were resolved through discussion with the third reviewer (Z.X.L).

\section{Quality assessment}

Two independent reviewers (Y.W.C and C.Y) evaluated the quality of the included studies by the NewcastleOttawa Scale [18], which was a nine-point scale that allocated points based on the selection process (0-4points), the comparability ( $0-2$ points), and the assessment of outcomes of study participants (0-3points). We assigned scores of $0-3,4-6$, and 7-9 for low, moderate, and high quality of studies, respectively.

\section{Statistical analysis}

Random-effects model was used to estimate the summary ORs or RRs for the association between hot beverage and food consumption and EC risk. Taking the subjectivity of differentiating between hot and very hot into account, we used the specific OR for standardized category (hot and very hot) versus reference category (cold and warm) of beverage and food consumption. We defined exposure as hot beverages and foods (standardized category, preference for high-temperature foods and drinks, often consuming of them) versus non-hot beverages and foods (all other combinations). If studies had partly overlapped subjects, only the one with a larger sample size was selected for the analysis. If a study reported results for different beverages and foods separately, those beverage/food specific results were regarded as separate reports on the relationship between temperature and EC risk. One study [11] contained 4 kinds of drinks, and was, therefore, accounted as four independent reports. Another study [19] reporting tea, water and food was regarded as three reports. Two studies [13,20] conducted in two different areas of China were considered as two reports respectively, and another study [16] including two large multicenter case-control studies was treated as two reports.

Statistical heterogeneity among studies was evaluated using the $I^{2}$ statistic, where values of 25\%, 50\% and 75\% represent cut-off points for low, moderate and high degrees of heterogeneity, respectively [21]. To assess the heterogeneity across all included studies, the study location (Asia, South America, Europe, Africa), study setting (populationbased, hospital based), study quality $(\geq 7,<7)$,type of EC (esophageal squamous cell carcinoma (ESCC), esophageal adenocarcinoma $($ EAC)), and sample size $(\geq 1000,<1000)$ were further examined using meta-regression. In sensitivity analyses, we conducted leave-one-out analyses [22] for each study to examine the magnitude of influence of each study on pooled risk estimates. Subgroup analyses by age, sex, study location, hot beverage and food categories, study 
quality, smoking and alcohol intake, study setting, outcome assessment and exposure assessment were conducted to examine the robustness of the primary results. Publication bias was assessed using the Begg test [23], the Egger test [24] and funnel plot. All statistical analyses were performed using STATA version 11.0 (Stata Corp, College Station, Texas, USA). All tests were two sided with a significance level of 0.05 .

\section{Results}

\section{Literature search}

The search identified a total of 3780 unique articles from PubMed, Google scholar, and Web of Science databases, of which 189 articles were identified as potentially relevant. After retrieving and reviewing the full text, we determined that 39 studies met our inclusion criteria. The process of study selection is shown in Figure 1.

\section{Study characteristics}

Table 1 shows the main characteristic of the 39 included studies. These studies were published between 1979 and 2014, all of which with case-control design. The sample sizes of studies ranged from 143 to 4,118 with a total of 42,475 subjects. The number of EC cases diagnosed in the studies ranged from 47 to 1,310, with a total of 13,811 reported EC cases. Seventeen studies were conducted in

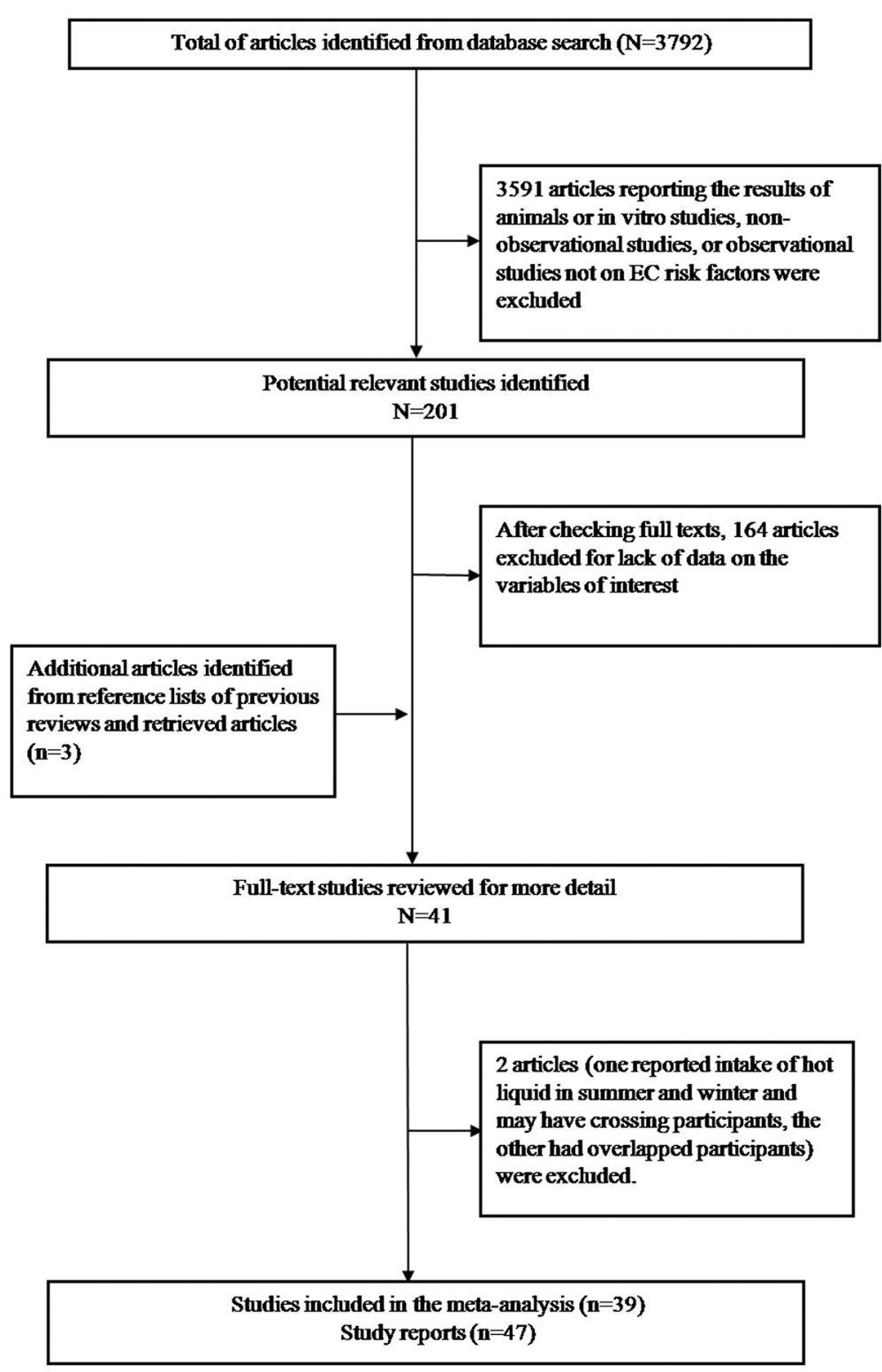

Figure 1 Study selection process. 
Table 1 Characteristics of studies included in the meta-analysis

Study source Study design Sex Age at No of No of

\begin{tabular}{clll} 
Exposure & Outcome assessment & $\begin{array}{l}\text { Exposure categories } \\
\text { used in meta-analysis }\end{array}$ \\
\hline Interviews & $\begin{array}{l}\text { Endoscopy and biopsy } \\
\text { samples }\end{array}$ & $\begin{array}{l}\text { Tea: hot/very } \\
\text { hot vs. warm }\end{array}$ \\
& &
\end{tabular}

\begin{tabular}{llll}
\hline Islami et al., & Population based & F/M & Cases:64.5 \pm 10.1 \\
[15], northern & case-control & & controls:64.3 \pm 10.4 \\
Iran & &
\end{tabular}

$300 \quad 871$

samples

hea: hot/very

Quality

assessment

Ethnicity, daily

vegetable intake,

alcohol consumption

tobacco or opium

use, duration of

residence in rura

areas, education level,

and car ownership

Lin et al

H5] Hospital based

China

F/M Cases:54.5 \pm 4.9

controls:52.5 \pm 3.7

Endoscopically and

histologically confirmed

hot vs. lukewarn

Age, sex, educational 6

status, smoking,

drinking, body mass

index, vegetable and

fruit

Rolon et al., Hospital based

[45], Paraguay case-control

F/M $\leq 45: 33$ 46-55:89

$131 \quad 512$

Interviews

$56-65: 188 \geq 66: 202$

Cytology, histology, or radiology

Maté: very hot vs.

warm/hot

design variables,

lifetime cigarette

consumption, and

lifetime alcoho

consumption

Stefani et al., Hospital based

[41], Uruguay case-control

Castelletto Hospital based

F/M 40-89

$166 \quad 830$

Questionnaire

Histologically verified

Maté: hot/very

hot vs, warm

Questionnaire

Histological diagnosis

Maté: hot/very

$\leq 54: 8055-64: 129$
$65-74: 127 \geq 75: 57$

Argentina

case-control

Castellsagu'e Hospital based

et al., [11],

south America

NR

Education, average number of cigarettes/ day, alcohol

consumption (ml/day), the design variables

Age group, hospital, 6

residency, years of

education, average
$830 \quad 2609$

F/M 64.0(mean)

F/M

structured

questionnaire

Histologically confirmed or a cytological or radiological diagnosis

Maté, tea, coffee, coffee with milk: hot/very hot vs. cold/warm

Registries

Tea/coffee: hot/very hot vs. lowest

$521 \quad 1965$

FFQ

number of cigarettes/

day, average amount

of pure ethanol/day

and gender

[12], Australia case-contro

Age, gender; cumulative 6

history of smoking in

pack years, lifetime

mean alcohol intake;

heartburn and acid

reflux symptoms, body

mass index, educationa

status, aspirin use in

previous 5 years, tota

fruit and vegetable

intake and total energy

intake in kilojoules 
Table 1 Characteristics of studies included in the meta-analysis (Continued)

\begin{tabular}{|c|c|c|c|c|c|c|c|c|c|c|}
\hline $\begin{array}{l}\text { Szyman'ska } \\
\text { et al., [44], } \\
\text { Latin America }\end{array}$ & $\begin{array}{l}\text { Hospital based } \\
\text { case-control }\end{array}$ & $\mathrm{F} / \mathrm{M}$ & NR & 71 & 228 & $\begin{array}{l}\text { Lifestyle } \\
\text { questionnaire }\end{array}$ & ICD-O classification & $\begin{array}{l}\text { Maté: hot/very } \\
\text { hot vs. cold/warm }\end{array}$ & NR & 5 \\
\hline $\begin{array}{l}\text { Chen et al., } \\
\text { [26], Southern } \\
\text { China }\end{array}$ & $\begin{array}{l}\text { Hospital based } \\
\text { case-control }\end{array}$ & $\mathrm{F} / \mathrm{M}$ & $\begin{array}{l}\text { Cases:54.6 } 6 \pm 6 \\
\text { controls: } 54.0 \pm 7\end{array}$ & 87 & 267 & $\begin{array}{l}\text { Self-designed } \\
\text { structured } \\
\text { questionnaire }\end{array}$ & Histologically confirmed & $\begin{array}{l}\text { Tea: hot/very } \\
\text { hot vs. warm }\end{array}$ & NR & 5 \\
\hline $\begin{array}{l}\text { Sewram et al., } \\
\text { [42], Uruguay }\end{array}$ & $\begin{array}{l}\text { Hospital based } \\
\text { case-control }\end{array}$ & $\mathrm{F} / \mathrm{M}$ & $35-85$ & 295 & 685 & Questionnaire & Registries & $\begin{array}{l}\text { Maté: very hot vs. } \\
\text { warm/hot }\end{array}$ & $\begin{array}{l}\text { Amount consumed, } \\
\text { and duration of mate' } \\
\text { consumption }\end{array}$ & 5 \\
\hline $\begin{array}{l}\text { Tang et al., } \\
\text { [19], China }\end{array}$ & $\begin{array}{l}\text { Hospital based } \\
\text { case-control }\end{array}$ & $\mathrm{F} / \mathrm{M}$ & $61 \pm 11.4$ & 359 & 739 & $\begin{array}{l}\text { Structured } \\
\text { questionnaire }\end{array}$ & $\begin{array}{l}\text { Medical records and } \\
\text { pathology reports }\end{array}$ & $\begin{array}{l}\text { Tea, water, food: } \\
\text { high vs. low or mild }\end{array}$ & $\begin{array}{l}\text { Age, gender, education } \\
\text { level, body mass index, } \\
\text { smoking status, alcohol } \\
\text { drinking, family history } \\
\text { of cancer in first-degree } \\
\text { relatives, daily intake of } \\
\text { vegetables and daily } \\
\text { intake of fruit }\end{array}$ & 6 \\
\hline $\begin{array}{l}\text { Stefani et al., } \\
\text { [40], Uruguay }\end{array}$ & $\begin{array}{l}\text { Hospital based } \\
\text { case-control }\end{array}$ & $\mathrm{F} / \mathrm{M}$ & $40-89$ & 234 & 702 & Questionnaire & $\begin{array}{l}\text { Microscopically } \\
\text { confirmed }\end{array}$ & $\begin{array}{l}\text { Maté: hot/very } \\
\text { hot vs. warm }\end{array}$ & NR & 5 \\
\hline $\begin{array}{l}\text { Wu et al., [20], } \\
\text { China }\end{array}$ & $\begin{array}{l}\text { Population based } \\
\text { case-control }\end{array}$ & $\mathrm{F} / \mathrm{M}$ & NR & 665 & 2000 & $\begin{array}{l}\text { Pretested } \\
\text { standardized } \\
\text { epidemiologic } \\
\text { questionnaire }\end{array}$ & registry & Tea: high vs. normal & NR & 5 \\
\hline $\begin{array}{l}\text { Sharp et al., } \\
\text { [8], England } \\
\text { and Scotland }\end{array}$ & $\begin{array}{l}\text { Population based } \\
\text { case-control }\end{array}$ & $\mathrm{F}$ & $<75$ (<80in Trent) & 156 & 312 & Interviews & Histologically confirmed & $\begin{array}{l}\text { Tea/coffee: hot/burning } \\
\text { hot vs. warm }\end{array}$ & NR & 5 \\
\hline $\begin{array}{l}\text { Terry et al., [7], } \\
\text { Sweden }\end{array}$ & $\begin{array}{l}\text { Population based } \\
\text { case-control }\end{array}$ & $\mathrm{F} / \mathrm{M}$ & $<80$ & 189 & 1004 & interviews & Histologically confirmed & $\begin{array}{l}\text { Tea/coffee: hot/very } \\
\text { hot vs. cold/lukewarm }\end{array}$ & $\begin{array}{l}\text { Age, gender, body mass } \\
\text { index, cigarette smoking, } \\
\text { socioeconomic status } \\
\text { presence of Gastro- } \\
\text { oesophageal reflux } \\
\text { symptoms, frequency } \\
\text { quartiles of hot } \\
\text { beverage consumption, } \\
\text { and quartiles of alcohol, } \\
\text { fruit and vegetables, and } \\
\text { energy consumption }\end{array}$ & 5 \\
\hline $\begin{array}{l}\text { Lubin et al., } \\
\text { 2014, South } \\
\text { America, [16] }\end{array}$ & Case-control & $\mathrm{F} / \mathrm{M}$ & $35-85$ & 1310 & 4118 & Questionnaire & Medical records & $\begin{array}{l}\text { Maté: hot/very hot vs. } \\
\text { warm vs. }\end{array}$ & NR & 5 \\
\hline $\begin{array}{l}\text { Wang et al. } \\
\text { [37], China }\end{array}$ & $\begin{array}{l}\text { Population based } \\
\text { case-control }\end{array}$ & $\mathrm{F} / \mathrm{M}$ & $\begin{array}{l}\text { Mean: cases } 61.51 \\
\text { controls } 60.75\end{array}$ & 355 & 763 & $\begin{array}{l}\text { Structured } \\
\text { questionnaire }\end{array}$ & $\begin{array}{l}\text { Pathologically } \\
\text { diagnosed }\end{array}$ & Food: hot vs. warm & $\begin{array}{l}\text { Age (continuous), } \\
\text { marital status and } \\
\text { education years }\end{array}$ & 7 \\
\hline
\end{tabular}


Table 1 Characteristics of studies included in the meta-analysis (Continued)

\begin{tabular}{|c|c|c|c|c|c|c|c|c|c|c|}
\hline $\begin{array}{l}\text { Phukan et al., } \\
\text { [47], India }\end{array}$ & $\begin{array}{l}\text { Hospital based } \\
\text { case-control }\end{array}$ & $\mathrm{F} / \mathrm{M}$ & $\begin{array}{l}\text { Case:55.0 } \pm 8.1 \\
\text { control:54.5 } \pm 7.8\end{array}$ & 502 & 1511 & Investigation & $\begin{array}{l}\text { Histopathologically } \\
\text { confirmed }\end{array}$ & Food: hot vs. moderate & $\begin{array}{l}\text { Education, income, } \\
\text { chewing betel nut } \\
\text { and tobacco, } \\
\text { smoking, and alcohol } \\
\text { use }\end{array}$ & 4 \\
\hline $\begin{array}{l}\text { Wu et al., [13], } \\
\text { China }\end{array}$ & $\begin{array}{l}\text { Population based } \\
\text { case-control }\end{array}$ & $\mathrm{F} / \mathrm{M}$ & $\begin{array}{l}<50: 6750-59: 219 \\
60-69: 42870- \\
79: 295 \geq 80: 53\end{array}$ & 531 & 1062 & $\begin{array}{l}\text { Pre-tested } \\
\text { standardized } \\
\text { questionnaires }\end{array}$ & $\begin{array}{l}\text { Cancer registration } \\
\text { database }\end{array}$ & Food: hot vs. normal & NR & 4 \\
\hline $\begin{array}{l}\text { Gao et al., [29], } \\
\text { China }\end{array}$ & $\begin{array}{l}\text { Population based } \\
\text { case-control }\end{array}$ & $\mathrm{F} / \mathrm{M}$ & $30-74$ & 902 & 2454 & $\begin{array}{l}\text { Structured } \\
\text { questionnaire }\end{array}$ & Registry & $\begin{array}{l}\text { Hot soup or porridge: } \\
\text { hot/burning hot vs. cold/ } \\
\text { neither cold nor hot }\end{array}$ & $\begin{array}{l}\text { Age, education, } \\
\text { birthplace, tea } \\
\text { drinking, cigarette } \\
\text { smoking, alcohol } \\
\text { drinking and } \\
\text { consumption of } \\
\text { preserved foods, } \\
\text { vegetables and fruit }\end{array}$ & 6 \\
\hline $\begin{array}{l}\text { Hu et al., [32], } \\
\text { China }\end{array}$ & $\begin{array}{l}\text { Hospital based } \\
\text { case-control }\end{array}$ & $\mathrm{F} / \mathrm{M}$ & $35-69$ & 196 & 588 & Interviews & $\begin{array}{l}\text { Histopathologically } \\
\text { confirmed }\end{array}$ & $\begin{array}{l}\text { Gruel: hot/scalding vs. } \\
\text { lowest }\end{array}$ & $\begin{array}{l}\text { Smoking, alcohol, } \\
\text { income and } \\
\text { occupation }\end{array}$ & 4 \\
\hline $\begin{array}{l}\text { Garidou et al., } \\
\text { [6], Greece }\end{array}$ & $\begin{array}{l}\text { Hospital based } \\
\text { case-control }\end{array}$ & $\mathrm{F} / \mathrm{M}$ & $\begin{array}{l}<60: 7960- \\
69: 103 \geq 70: 117\end{array}$ & 99 & 299 & Questionnaire & Histologically confirmed & $\begin{array}{l}\text { Preferrable temperature: } \\
\text { very hot vs. cold to hot }\end{array}$ & $\begin{array}{l}\text { Gender, age, } \\
\text { birthplace, schooling, } \\
\text { height, analgesics, } \\
\text { coffee drinking, } \\
\text { alcohol intake, } \\
\text { tobacco smoking and } \\
\text { energy intake }\end{array}$ & 4 \\
\hline $\begin{array}{l}\text { Cheng et al., } \\
\text { [51], British }\end{array}$ & $\begin{array}{l}\text { Population based } \\
\text { case-control }\end{array}$ & $\mathrm{F}$ & $\begin{array}{l}\text { Cases:65.9 } \\
\text { controls:65.3 }\end{array}$ & 74 & 148 & $\begin{array}{l}\text { Questionnaire and } \\
\text { interview }\end{array}$ & Histologically confirmed & $\begin{array}{l}\text { Preference tea or coffee: } \\
\text { hot very/burning hot/hot } \\
\text { vs. warm }\end{array}$ & NR & 4 \\
\hline $\begin{array}{l}\text { Hanaoka et al., } \\
\text { [53], Japan }\end{array}$ & $\begin{array}{l}\text { Hospital based } \\
\text { case-control }\end{array}$ & M & Under 85 years old & 141 & 282 & $\begin{array}{l}\text { Structured } \\
\text { questionnaire }\end{array}$ & $\begin{array}{l}\text { Confirmed histologically } \\
\text { by biopsy examination }\end{array}$ & $\begin{array}{l}\text { Preference for high }= \\
\text { temperature food and } \\
\text { drink: like vs. dislike }\end{array}$ & $\begin{array}{l}\text { Alcohol consumption } \\
\text { (g/week) }\end{array}$ & 4 \\
\hline $\begin{array}{l}\text { Srivastava } \\
\text { et al., [48], } \\
\text { India }\end{array}$ & Case-control & $\mathrm{F} / \mathrm{M}$ & NR & 170 & 340 & $\begin{array}{l}\text { Pretested. Semi- } \\
\text { structured } \\
\text { questionnaires }\end{array}$ & $\begin{array}{l}\text { Endoscopic, radiological } \\
\text { and histopathological } \\
\text { assessments }\end{array}$ & Food: hot vs. warm & NR & 4 \\
\hline $\begin{array}{l}\text { Stefani et al., } \\
\text { [39], Uruguay }\end{array}$ & $\begin{array}{l}\text { Hospital based } \\
\text { case-control }\end{array}$ & $\mathrm{F} / \mathrm{M}$ & $\begin{array}{l}40-49: 45 \text { 50-59:120 } \\
60-69: 20770- \\
79: 183 \text { 80-89:45 }\end{array}$ & 200 & 600 & Questionnaire & $\begin{array}{l}\text { Newly diagnosed and } \\
\text { microspically confirmed }\end{array}$ & $\begin{array}{l}\text { Maté temperature: hot/ } \\
\text { very hot vs. warm }\end{array}$ & NR & 5 \\
\hline $\begin{array}{l}\text { Cheng et al., } \\
\text { [27], Hong } \\
\text { Kong of China }\end{array}$ & Case-control & $\mathrm{F} / \mathrm{M}$ & $\begin{array}{l}<45: 40 \text { 45-54:246 } \\
55-64: 722 \text { 65- } \\
74: 696>=75: 294\end{array}$ & 400 & 1998 & $\begin{array}{l}\text { Interviews with } \\
\text { structured } \\
\text { questionnaire }\end{array}$ & $\begin{array}{l}\text { Histologically confirmed } \\
\text { diagnoses }\end{array}$ & $\begin{array}{l}\text { Preference for hot drinks } \\
\text { or soups: yes vs. no }\end{array}$ & $\begin{array}{l}\text { Adjusted for age and } \\
\text { education, place of } \\
\text { birth, green leafy } \\
\text { vegetables, pickled } \\
\text { vegetables, citrus } \\
\text { fruits, tobacco and } \\
\text { alcohol }\end{array}$ & 4 \\
\hline
\end{tabular}


Table 1 Characteristics of studies included in the meta-analysis (Continued)

\begin{tabular}{|c|c|c|c|c|c|c|c|c|c|c|}
\hline $\begin{array}{l}\text { Gao et al., [30], } \\
\text { China }\end{array}$ & $\begin{array}{l}\text { Population based } \\
\text { case-control }\end{array}$ & $\mathrm{F} / \mathrm{M}$ & $30-74$ & 653 & 1965 & $\begin{array}{l}\text { Structured, } \\
\text { standardized } \\
\text { questionnaire }\end{array}$ & Registry & $\begin{array}{l}\text { Burning-hot fluids:yes vs. } \\
\text { no }\end{array}$ & NR & 5 \\
\hline $\begin{array}{l}\text { Cook- } \\
\text { mozaffari } \\
\text { et al., [49], Iran }\end{array}$ & Case-control & $\mathrm{F} / \mathrm{M}$ & NR & 344 & 1032 & Questionnaire & Registry & $\begin{array}{l}\text { Drinking of hot tea: yes } \\
\text { vs. no }\end{array}$ & NR & 4 \\
\hline $\begin{array}{l}\text { Guo et al.,[31], } \\
\text { China }\end{array}$ & $\begin{array}{l}\text { Nested case- } \\
\text { control }\end{array}$ & $\mathrm{F} / \mathrm{M}$ & $40-69$ & 640 & 3840 & $\begin{array}{l}\text { Structured } \\
\text { questionnaires }\end{array}$ & $\begin{array}{l}\text { X-ray films and } \\
\text { cytological, pathological, } \\
\text { surgical specimens }\end{array}$ & Hot liquid: $\geq 1$ vs. 0 & $\begin{array}{l}\text { Years of smoking and } \\
\text { cancer history in first } \\
\text { degree relatives }\end{array}$ & 6 \\
\hline $\begin{array}{l}\text { Ke et al., [34], } \\
\text { China }\end{array}$ & $\begin{array}{l}\text { Hospital based } \\
\text { case-control }\end{array}$ & $\mathrm{F} / \mathrm{M}$ & $29-82$ & 1064 & 2168 & $\begin{array}{l}\text { Questionnaires and } \\
\text { FFQ }\end{array}$ & Histologically confirmed & $\begin{array}{l}\text { Hot Congou drinkers vs. } \\
\text { non-hot Congou drinkers }\end{array}$ & NR & 5 \\
\hline $\begin{array}{l}\text { Patel et al., } \\
\text { [52], Kenya }\end{array}$ & $\begin{array}{l}\text { Hospital based } \\
\text { case-control }\end{array}$ & $\mathrm{F} / \mathrm{M}$ & Mean:56.1 & 159 & 318 & Questionnaires & NR & $\begin{array}{l}\text { Take hot beverages: yes/ } \\
\text { no }\end{array}$ & NR & 4 \\
\hline $\begin{array}{l}\text { Hung et al., } \\
\text { [33], Taiwan of } \\
\text { China }\end{array}$ & Case-control & M & Mean:62.4 & 267 & 697 & $\begin{array}{l}\text { Interviews } \\
\text { according to } \\
\text { standardized } \\
\text { questionnaire }\end{array}$ & Histologically confirmed & $\begin{array}{l}\text { Hot drink or soup: } 3+ \\
\text { time per day vs. }<3 \text { time } \\
\text { per day }\end{array}$ & $\begin{array}{l}\text { Adjusted for age, } \\
\text { educational levels, } \\
\text { ethnicity, source of } \\
\text { hospital, smoking, } \\
\text { alcohol drinking and } \\
\text { areca nut chewing }\end{array}$ & 4 \\
\hline $\begin{array}{l}\text { Chen et al., } \\
\text { [25], Taiwan of } \\
\text { China }\end{array}$ & $\begin{array}{l}\text { Hospital based } \\
\text { case-control }\end{array}$ & M & $\begin{array}{l}40-50: 28451- \\
60: 29161-70 \\
: 314>70: 209\end{array}$ & 274 & 922 & Interviews & $\begin{array}{l}\text { Newly histologically } \\
\text { diagnosed }\end{array}$ & $\begin{array}{l}\text { Hot drink or soup: }>=1 \\
\text { time/d vs. <1time/d }\end{array}$ & $\begin{array}{l}\text { Adjusted for age, } \\
\text { educational levels, } \\
\text { ethnicity, source of } \\
\text { hospital, smoking, } \\
\text { alcohol drinking, and } \\
\text { areca nut chewing }\end{array}$ & 4 \\
\hline $\begin{array}{l}\text { Gao et al., } \\
\text { [28],China }\end{array}$ & Case-control & $\mathrm{F} / \mathrm{M}$ & $51-65$ & 600 & 2114 & Questionnaires & Histologically confirmed & $\begin{array}{l}\text { Scalding hot food: daily } \\
\text { vs. weekly/never/ } \\
\text { monthly/seldom }\end{array}$ & NR & 4 \\
\hline $\begin{array}{l}\text { Sun et al., } \\
\text { [36],China }\end{array}$ & $\begin{array}{l}\text { Population based } \\
\text { case-control }\end{array}$ & $\mathrm{F} / \mathrm{M}$ & $\begin{array}{l}\text { Cases: } 61.21 \pm 8.95 \\
\text { Controls: } 60.84 \pm \\
8.90\end{array}$ & 250 & 1000 & Questionnaires & $\begin{array}{l}\text { Cancer registration } \\
\text { database }\end{array}$ & $\begin{array}{l}\text { Hot foods: often vs. } \\
\text { sometimes }\end{array}$ & NR & 6 \\
\hline $\begin{array}{l}\text { Yang et al., } \\
\text { [38], China }\end{array}$ & Case-control & $\mathrm{F} / \mathrm{M}$ & $\begin{array}{l}\text { Cases:58.1 (8.5) } \\
\text { Controls:57.9 (8.8) }\end{array}$ & 185 & 370 & Questionnaires & $\begin{array}{l}\text { Histologically diagnosed } \\
\text { within half a year }\end{array}$ & $\begin{array}{l}\text { Hot foods: often vs. } \\
\text { Rarely/occasionally }\end{array}$ & NR & 6 \\
\hline $\begin{array}{l}\text { Jessri et al., } \\
\text { [50], Iran }\end{array}$ & $\begin{array}{l}\text { Hospital based } \\
\text { case-control }\end{array}$ & $\mathrm{F} / \mathrm{M}$ & $40-75$ & 47 & 143 & $\begin{array}{l}\text { Structured pre- } \\
\text { tested } \\
\text { questionnaires }\end{array}$ & Histologically-confirmed & $\begin{array}{l}\text { Food and beverages } \\
\text { temperature: hot vs. } \\
\text { warm/cold }\end{array}$ & NR & 4 \\
\hline $\begin{array}{l}\text { Khan et al., } \\
\text { [46], India }\end{array}$ & Case-control & $\mathrm{F} / \mathrm{M}$ & $\begin{array}{l}\text { Case:54.3(7.6) } \\
\text { Control:58.1(8.3) }\end{array}$ & 100 & 200 & Questionnaires & Histologically-confirmed & $\begin{array}{l}\text { Degree of hotness: hot } \\
\text { vs. warm }\end{array}$ & NR & 3 \\
\hline
\end{tabular}

Age presents the range with Mean (SD). Abbreviations: $N R=$ not reported; $F=$ female; $M=$ male 
China [13,19,20,25-38], six in Uruguay [11,16,39-42], three in Argentina [11,16,43], three in Brazil $[11,16,44]$, three in Paraguay $[11,16,45]$, three in India [46-48], three in Iran [15,49,50], two in British [8,51], one in Australia [12], one in Sweden [7], one in Greece [6], one in Kenya [52], and one in Japan [53]. Thirty studies reported results for men and women together, four reported the results for men and women separately, and three reported results for men only and two for women only. Three studies reported results separately by type of EC. Two studies were deemed high quality, 36 moderate quality studies, and one low quality study. The average quality score for all included studies was 5.00 .

\section{Hot beverage and food consumption and the risk of esophageal cancer}

The results from the random-effects meta-analysis of hot beverage and food consumption and the risk of EC were shown in Figure 2. Thirty-two of 47 independent reports from 39 studies suggested a positive relation between hot beverage and food consumption and EC risk. The pooled OR was $1.77(95 \% \mathrm{CI}, 1.39-2.25)$, with a high heterogeneity $\left(I^{2}=92.8 \%, p=0.001\right)$; the pooled OR was $2.09(95 \% \mathrm{CI}$, $\left.1.71-2.56, I^{2}=57.8 \%, p=0.008\right)$; and the pooled OR of EC risk in relation to hot beverage and food consumption was 1.73(95\% CI, $\left.1.18-2.53, I^{2}=68.2 \%, p=0.004\right)$.

\section{Subgroup analysis}

Table 2 showed the results based on subgroup analyses, which were to examine the stability of the primary results and explore the resources of potential heterogeneity. The associations between hot beverage and food consumption and the risk of EC were similarly significant in subgroup analyses, with the exception of EAC $\left(\mathrm{OR}=0.79,95 \% \mathrm{CI}=0.53-1.16, I^{2}=50.30 \%, P=0.110\right)$ and European population $(\mathrm{OR}=0.95,95 \% \mathrm{CI}=0.68-1.34$, $I^{2}=62.40 \%, P=0.031$.

\section{Sensitivity analysis and meta-regression}

We excluded each study in turn and pooled the results of the remaining included studies. The positive association was not materially changed upon the exclusions, with a pooled OR range from 1.75 (95\% $\mathrm{CI}, 1.47$ to $2.07 ; P=$ 0.001 ) to $1.87(95 \% \mathrm{CI}, 1.58$ to $2.20 ; P=0.001$ ), which indicates that the overall result was not significantly influenced by any individual studies.

Our meta-regression analysis reveals that the study location $(P=0.001)$, the type of EC $(P=0.047)$ and sample size $(P=0.033)$ were significant sources of heterogeneity. Study location alone explained $34.39 \%$ of the $\tau^{2}$ in the meta-regression; type of EC explained 12.97\%; and sample size explained $8.99 \%$. The results were shown in Table 3.

\section{Publication bias}

Visual inspection of funnel plot did not identify substantial asymmetry (see Figure 3). The Begg rank correlation test and the Egger linear regression test indicated no evidence of publication bias across included studies (Begg test $Z=0.59, P=0.557$; Egger test $t=1.58, P=0.121$ ).

\section{Discussion}

In this large pooled analysis of 42475 participants (13811 EC cases) from 39 case-control studies, we confirmed a positive association between hot beverage and food consumption and EC risk. Individuals who usually have beverages and food served very hot or hot were almost twice likely to develop EC than individuals who usually have beverages and foods served warm or cold. Our subgroup analyses show that the results held true across various populations despite significant heterogeneity.

Our meta-analysis shows that the consumption of hot beverages and foods are significantly associated with ESCC (OR, 1.60; 95\% CI, 1.29-2.00) but not with EAC (0.79, 95\% CI, 0.53-1.16). A large body of observational evidence suggests that the risk factors for ESCC and EAC may be different. For example, alcohol intake is a strong and well established risk factor for ESCC but it is not associated with EAC [54]; a high body mass index (BMI) is associated with an increased risk of EAC but a decreased risk of ESCC [55]; ESCC is strongly associated with high-level exposure to tobacco smoking in Western populations [54,56], whereas EAC is associated with gastro-esophageal reflux disease and Barrett's esophagus [57]. More studies are needed to explore why hot beverage and food consumption is associated with an increased risk for ESCC but not EAC.

Another notable finding is that hot beverage and food consumption appears not to be a risk factor for EC in European population (OR, 0.95; 95\% CI, 0.68-1.34). The result might be ascribed to the small sample size (3,728 participants and 1,039 EC cases) or the unique dietary habits of Europeans. A previous study noted that Europeans tend to add cold milk to the exposure beverages, tea or coffee before consumption [12], which may cause people say they drink hot actually only warm and result in substantial difference between the temperature perceived by drinkers and the actual temperature of their drinks.

It is conceivable that hot beverages and foods may cause thermal injury to the esophageal mucosa, and there are several biological mechanisms through which thermal injury in general could increase the risk of EC. Inflammatory processes associated with chronic irritation of the esophageal mucosa caused by local hyperthermia could stimulate the endogenous formation of reactive nitrogen species and nitrosamines [58]. This hypothesis is supported by a high rate of somatic $\mathrm{G}$ to $\mathrm{A}$ transitions in CpG dinucleotides of the TP53 gene in 


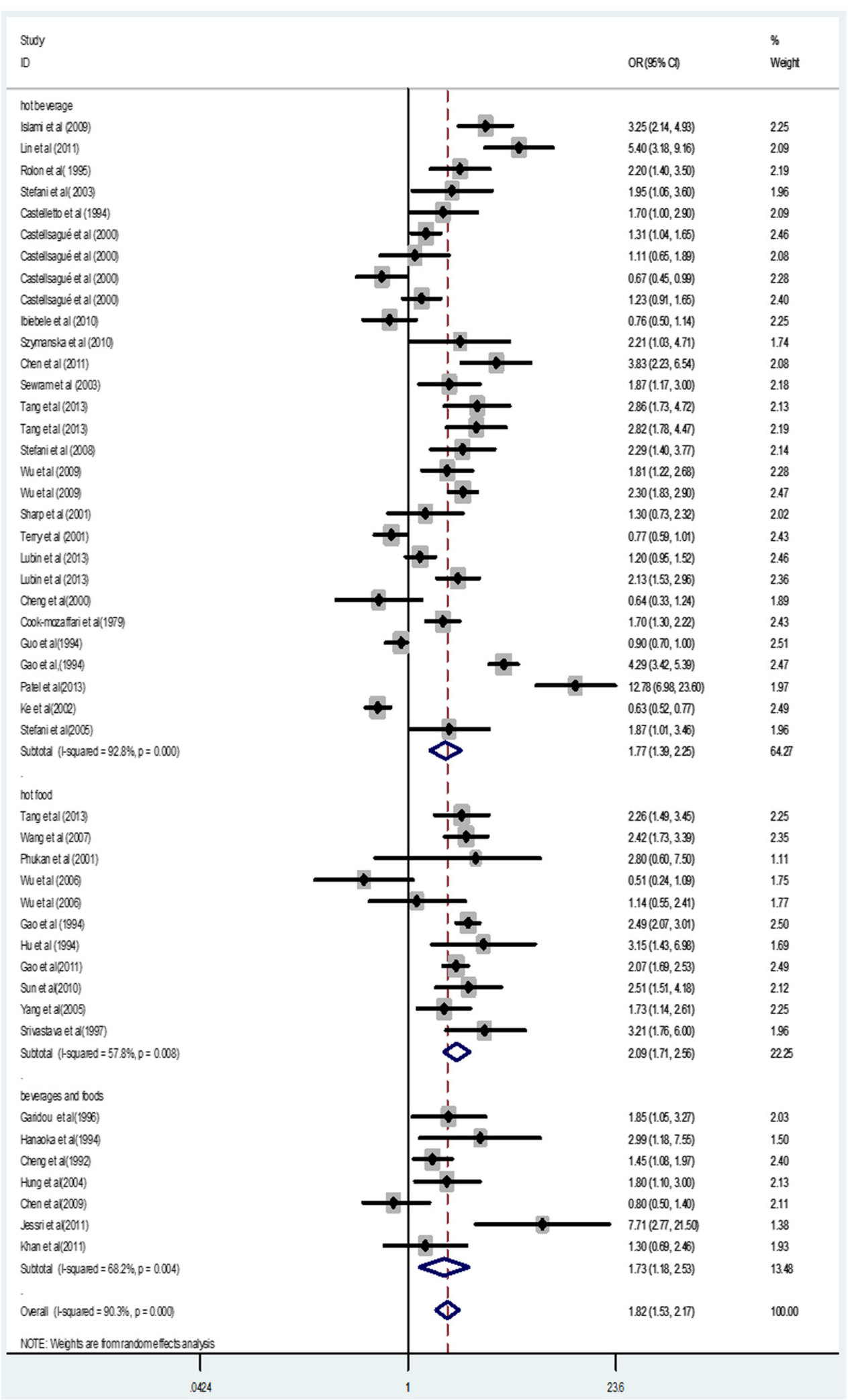

Figure 2 Forest plot of odds ratios from 39 studies linking hot beverage and food consumption and the risk of esophageal cancer. 
Table 2 Subgroup analysis of odds ratio of hot beverages and foods and esophageal cancer

\begin{tabular}{|c|c|c|c|c|c|}
\hline & $\begin{array}{l}\text { No of } \\
\text { reports }\end{array}$ & OR & $(95 \% \mathrm{Cl})$ & $1^{2}$ & $\begin{array}{l}\mathrm{P} \text { for } \\
\text { heterogeneity }\end{array}$ \\
\hline \multicolumn{6}{|l|}{$\overline{\text { Sex }}$} \\
\hline Men & 8 & 2.36 & $1.53-3.65$ & $87.60 \%$ & 0.001 \\
\hline Women & 7 & 2.45 & $1.51-3.98$ & $85.60 \%$ & 0.001 \\
\hline Combined & 37 & 1.78 & $1.49-2.16$ & $89.30 \%$ & 0.001 \\
\hline \multicolumn{6}{|l|}{ Type of EC } \\
\hline ESCC & 26 & 1.60 & $1.29-2.00$ & $88.70 \%$ & 0.001 \\
\hline EAC & 4 & 0.79 & $0.53-1.16$ & $50.30 \%$ & 0.110 \\
\hline$N R$ & 20 & 2.35 & $1.90-2.91$ & $80.70 \%$ & 0.001 \\
\hline \multicolumn{6}{|l|}{ Study quality } \\
\hline Score $\geq 7$ & 2 & 2.73 & $2.06-3.62$ & $12.90 \%$ & 0.284 \\
\hline Score $<7$ & 45 & 1.78 & $1.49-2.14$ & $90.40 \%$ & 0.001 \\
\hline \multicolumn{6}{|l|}{ Study location } \\
\hline Asia & 28 & 2.06 & $1.62-2.61$ & $91.70 \%$ & 0.001 \\
\hline South America & 13 & 1.52 & $1.25-1.85$ & $66.70 \%$ & 0.001 \\
\hline Europe & 5 & 0.95 & $0.68-1.34$ & $62.40 \%$ & 0.031 \\
\hline Africa & 1 & 12.78 & $6.95-23.5$ & . & 0.001 \\
\hline
\end{tabular}

Measurement

domain

Temperature categories

Whether

consuming or not

Preference

Frequency

Beverages and

foods domain

Tea

Mate

Foods

Others

Controlling age in models

Yes

No

Controlling

smoking in models

Yes

No

$\begin{array}{llll}1.61 & 1.26-2.07 \quad 89.30 \% & 0.001\end{array}$

$2 \quad 1.56-2.55 \quad 90.50 \% \quad 0.001$

Controlling alcohol intake in models

$\begin{array}{lccccc}\text { Yes } & 19 & 1.56 & 1.21-2.02 & 88.00 \% & 0.001 \\ \text { No } & 28 & 2.03 & 1.59-2.59 & 91.60 \% & 0.001 \\ \text { Study setting } & & & & & \\ \text { Population } & 14 & 1.52 & 1.07-2.16 & 94.1 \% & 0.001 \\ \text { Hospital } & 24 & 2.10 & 1.56-2.82 & 89.8 \% & 0.001\end{array}$

Table 2 Subgroup analysis of odds ratio of hot beverages and foods and esophageal cancer (Continued)

\begin{tabular}{lccccc}
\hline NR & 9 & 1.73 & $1.44-2.06$ & $59.7 \%$ & 0.040 \\
$\begin{array}{l}\text { Exposure } \\
\text { assessment }\end{array}$ & & & & & \\
Interview & 14 & 1.33 & $1.03-1.71$ & $80.0 \%$ & 0.001 \\
Questionnaire & 33 & 2.07 & $1.67-2.57$ & $91.5 \%$ & 0.001 \\
$\begin{array}{l}\text { Outcome } \\
\text { assessment }\end{array}$ & & & & & \\
Histology & 30 & 1.68 & $1.36-2.07$ & $88.2 \%$ & 0.001 \\
Record & 17 & 1.90 & $1.50-2.41$ & $86.3 \%$ & 0.001
\end{tabular}

Abbreviations: $\mathrm{EC}=$ esophageal cancer; $\mathrm{ESCC}=$ esophageal squamous cell carcinoma; $\mathrm{EAC}=$ esophageal adenocarcinoma; $\mathrm{NR}=$ not reported.

esophageal tumor samples from geographical areas in which drinking hot beverages is considered an important risk factor for EC [59-62]; these mutations may indicate increased nitric oxide synthase activity in tumors [63]. The barrier function of the esophageal epithelium can be impaired by thermal injury, which may increase the risk of damage from exposure to intraluminal carcinogens [10], such as polycyclic aromatic hydrocarbons. Elevated temperatures could also accelerate metabolic reaction, including those with carcinogenic substances in tobacco and alcohol [64]. In fact, the association between consuming hot drinks and the occurrence of precancerous lesions of the esophagus has been repeatedly reported [65-67]. In addition, dietary deficiencies may weaken the esophageal tissue because of the constant irritation, which may act as a predisposing factor for EC [47]. It has also been postulated that contact of hot liquid and food with the esophageal mucosa could increase gastric reflux, causing further damage from gastric acid [68]. One review proposed that the overproduction of prostaglandin $E_{2}$ and leukotriene $\mathrm{B}_{4}$ as well as overexpression of their receptors are major factors in exacerbating inflammation and oxidative stress, which is the main pathogenesis associated with EAC [57]. The result from our meta-analysis of epidemiological studies is consistent with these biomedical research findings and postulations.

Table 3 Meta-regression analysis

\begin{tabular}{lllll}
\hline Variable & Coefficient & Standard error & $\boldsymbol{P}$ value & $\mathbf{9 5 \%} \mathbf{C l}$ \\
\hline Study location & & & & \\
Asia & -1.833 & 0.568 & 0.002 & $-2.979--0.688$ \\
South America & -2.109 & 0.578 & 0.001 & $-3.273--0.945$ \\
Europe & -2.582 & 0.607 & 0.001 & $-3.807--1.357$ \\
Type of EC & -0.678 & 0.327 & 0.047 & $-1.348--0.009$ \\
Sample size & -0.403 & 0.183 & 0.033 & $-0.771--0.034$ \\
\hline Abbreviations: EC $=$ esophageal cancer. & &
\end{tabular}




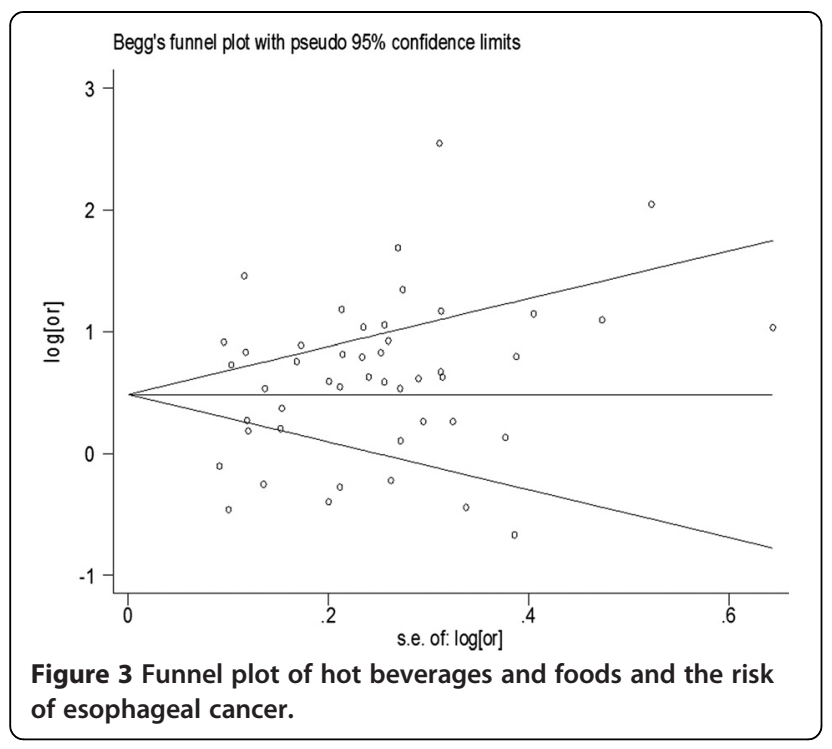

All the original studies used in our meta-analysis are of case-control study design, which is particularly vulnerable to potential biases (both selection bias and information bias). The included studies were conducted among different populations, mostly along with various categorizations of beverages and foods, which could confound our analysis on the specific link between the temperature of beverages and foods and the risk of EC. Lastly, the study relied on self-reported consumption of hot beverages and foods; as a result, the categorization of "hot or very hot" versus "cold or warm" is subject to reporting bias. In addition, the limited information provided in the included studies ruled out the possibility of conducting a doseresponse analysis. Nonetheless, this is the first metaanalysis to systematically quantify the association between hot beverage and food consumption and EC risk, and the results of our study are of broad interest to medical science and the public since consumption of beverages such as tea, coffee, and maté are prevalent worldwide $[64,69,70]$ and many people prefer to drink them at a high or very high temperature [15,71].

In the light of our findings, certain factors should be considered in future studies. Large prospective studies are needed to investigate the association of hot beverage and food consumption with both EC risk and the type of EC, not only because of the different ESCC and EAC risk factors but also the rapid changes in incidence of EAC [12]. In addition, measuring the actual temperature of hot beverage and food would provide dose-response data that would allow for evaluation of the relationship with EC risk more precisely. Finally, confounding factors, such as BMI, smoking, alcohol intake, and socioeconomic status, should be adjusted to allow dissection of the actual influence of hot beverage and food on EC, thereby providing provide stronger research-based evidence.

\section{Conclusions}

In summary, our meta-analysis shows that hot beverage and food consumption is associated with a significantly increase in the risk of EC, especially in Asian and South American populations. Given that hot beverages and foods are prevalent in modern society, the results of our meta-analysis have important implications for cancer etiology research as well as applications in health education and clinical practice.

\section{Ethics approval}

Ethical approval is not required for this review.

\section{Competing interests}

The authors declare that they have no competing interests.

\section{Authors' contributions}

YWC, CY, SYC and ZXL designed the study. YWC, CY, HLS and HSB carried out the data collection. YWC, CY, YQT and YG performed the statistical analyses. YWC, CY, GY, HLS and XXY drafted the manuscript. All authors read and approved the final manuscript.

\section{Acknowledgements}

We thank all the authors of the studies included in our meta-analysis.

\section{Funding}

No funding was received for this systematic review.

\section{Author details}

${ }^{1}$ School of Public Health, Tongji Medical College, Huazhong University of Science and Technology, Wuhan, Hubei, China. ${ }^{2}$ Center for Disease Control and Prevention of Hubei Province, Wuhan, Hubei, China.

Received: 25 July 2014 Accepted: 12 March 2015

Published online: 02 June 2015

\section{References}

1. Kamangar F, Dores GM, Anderson WF. Patterns of cancer incidence, mortality, and prevalence across five continents: defining priorities to reduce cancer disparities in different geographic regions of the world. J Clin Oncol. 2006;24(14):2137-50.

2. Andrici J, Eslick GD. Mate consumption and the risk of esophageal squamous cell carcinoma: a meta-analysis. Dis Esophagus. 2013;26(8):807-16.

3. Yu X, Bao Z, Zou J, Dong J. Coffee consumption and risk of cancers: a meta-analysis of cohort studies. BMC Cancer. 2011;11:96.

4. Morse MA, Kresty LA, Steele VE, Kelloff GJ, Boone CW, Balentine DA, et al. Effects of theaflavins on $\mathrm{N}$-nitrosomethylbenzylamine-induced esophageal tumorigenesis. Nutr Cancer. 1997:29(1):7-12.

5. Candreva EC, Keszenman DJ, Barrios E, Gelos U, Nunes E. Mutagenicity induced by hyperthermia, hot mate infusion, and hot caffeine in Saccharomyces cerevisiae. Cancer Res. 1993;53(23):5750-3.

6. Garidou A, Tzonou A, Lipworth L, Signorello LB, Kalapothaki V, Trichopoulos D. Life-style factors and medical conditions in relation to esophageal cancer by histologic type in a low-risk population. Int J Cancer. 1996;68(3):295-9.

7. Terry JL P, Wolk A, Nyrén O. Drinking hot beverages is not associated with risk of oesophageal cancers in a Western population. Br J Cancer. 2001:84:120-1.

8. Sharp L, Chilvers CE, Cheng KK, McKinney PA, Logan RF, Cook-Mozaffari P, et al. Risk factors for squamous cell carcinoma of the oesophagus in women: a case-control study. Br J Cancer. 2001;85(11):1667-70.

9. Islami F, Boffetta P, Ren J-S, Pedoeim L, Khatib D, Kamangar F. High-temperature beverages and foods and esophageal cancer risk-A systematic review. Int J Cancer. 2009:125(3):491-524.

10. Tobey NA, Sikka D, Marten E, Caymaz-Bor C, Hosseini SS, Orlando RC. Effect of heat stress on rabbit esophageal epithelium. Am J Physiol. 1999;276(6 Pt 1):G1322-1330. 
11. Castellsague X, Munoz N, De Stefani E, Victora CG, Castelletto R, Rolon PA. Influence of mate drinking, hot beverages and diet on esophageal cancer risk in South America. Int J Cancer. 2000;88(4):658-64.

12. Ibiebele TI, Taylor AR, Whiteman DC, van der Pols JC. Eating habits and risk of esophageal cancers: a population-based case-control study. Cancer Causes Control. 2010:21(9):1475-84

13. Wu M, Zhao JK, Hu XS, Wang PH, Qin Y, Lu YC, et al. Association of smoking, alcohol drinking and dietary factors with esophageal cancer in high- and lowrisk areas of Jiangsu Province, China. World J Gastroenterol. 2006;12(11):1686-93.

14. Steiner PE. The etiology and histogenesis of carcinoma of the esophagus. Cancer. 1956;9(3):436-52

15. Islami F, Pourshams A, Nasrollahzadeh D, Kamangar F, Fahimi S, Shakeri R, et al. Tea drinking habits and oesophageal cancer in a high risk area in northern Iran: population based case-control study. BMJ. 2009:338:b929.

16. Lubin JH, De Stefani E, Abnet CC, Acosta G, Boffetta P, Victora C, et al. Mate drinking and esophageal squamous cell carcinoma in South America: pooled results from two large multicenter case-control studies. Cancer Epidemiol Biomarkers Prev. 2014;23(1):107-16.

17. Stroup DF, Berlin JA, Morton SC, Olkin I, Williamson GD, Rennie D, et al. Meta-analysis of observational studies in epidemiology: a proposal for reporting. Meta-analysis Of Observational Studies in Epidemiology (MOOSE) group. JAMA. 2000;283(15):2008-12.

18. Wells G SB OCD, Peterson J, Welch V, Losos M. The Newcastle - Scale for assessing the quality of nonrandomised studies in meta-analyses. http://www.ohri.ca/programs/clinical_epidemiology/oxford.htm

19. Tang L, Xu F, Zhang T, Lei J, Binns CW, Lee AH. High temperature of food and beverage intake increases the risk of oesophageal cancer in Xinjiang, China. Asian Pac J Cancer Prev. 2013;14(9):5085-8.

20. Wu M, Liu AM, Kampman E, Zhang ZF, Van't Veer P, Wu DL, et al. Green tea drinking, high tea temperature and esophageal cancer in high- and low-risk areas of Jiangsu Province, China: a population-based case-control study. Int J Cancer. 2009;124(8):1907-13.

21. Higgins JP, Thompson SG. Quantifying heterogeneity in a meta-analysis. Stat Med. 2002:21(11):1539-58.

22. Wallace BC, Schmid CH, Lau J, Trikalinos TA. Meta-analyst: software for meta-analysis of binary, continuous and diagnostic data. BMC Med Res Methodol. 2009;9:80

23. Begg CB, Mazumdar M. Operating characteristics of a rank correlation test for publication bias. Biometrics. 1994;50(4):1088-101.

24. Egger M, Davey Smith G, Schneider M, Minder C. Bias in meta-analysis detected by a simple, graphical test. BMJ. 1997;315(7109):629-34.

25. Chen YK, Lee CH, Wu IC, Liu JS, Wu DC, Lee JM, et al. Food intake and the occurrence of squamous cell carcinoma in different sections of the esophagus in Taiwanese men. Nutrition. 2009;25(7-8):753-61.

26. Chen Z, Chen Q, Xia H, Lin J. Green tea drinking habits and esophageal cancer in southern China: a case-control study. Asian Pac J Cancer Prev. 2011;12(1):229-33.

27. Cheng KK, Day NE, Duffy SW, Lam TH, Fok M, Wong J. Pickled vegetables in the aetiology of oesophageal cancer in Hong Kong Chinese. Lancet. 1992;339(8805):1314-8

28. Gao Y, Hu N, Han XY, Ding T, Giffen C, Goldstein AM, et al. Risk factors for esophageal and gastric cancers in Shanxi Province, China: a case-control study. Cancer Epidemiol. 2011;35(6):e91-99.

29. Gao YT, McLaughlin JK, Blot WJ, Ji BT, Dai Q, Fraumeni Jr JF. Reduced risk of esophageal cancer associated with green tea consumption. J Natl Cancer Inst. 1994;86(11):855-8

30. Gao YT, McLaughlin JK, Gridley G, Blot WJ, Ji BT, Dai Q, et al. Risk factors for esophageal cancer in Shanghai, China. II. Role of diet and nutrients. Int J Cancer. 1994;58(2):197-202.

31. Guo W, Blot WJ, Li JY, Taylor PR, Liu BQ, Wang W, et al. A nested casecontrol study of oesophageal and stomach cancers in the Linxian nutrition intervention trial. Int J Epidemiol. 1994;23(3):444-50.

32. Hu J, Nyren O, Wolk A, Bergstrom R, Yuen J, Adami HO, et al. Risk factors for oesophageal cancer in northeast China. Int J Cancer. 1994;57(1):38-46.

33. Hung HC, Huang MC, Lee JM, Wu DC, Hsu HK, Wu MT. Association between diet and esophageal cancer in Taiwan. J Gastroenterol Hepatol. 2004;19(6):632-7.

34. Ke L, Yu P, Zhang ZX, Huang SS, Huang G, Ma XH. Congou tea drinking and oesophageal cancer in South China. Br J Cancer. 2002;86(3):346-7.

35. Lin J, Zeng R, Cao W, Luo R, Chen J, Lin Y. Hot beverage and food intake and esophageal cancer in southern China. Asian Pac J Cancer Prev. 2011;12(9):2189-92.
36. Sun X, Chen W, Chen Z, Wen D, Zhao D, He Y. Population-based case-control study on risk factors for esophageal cancer in five high-risk areas in China. Asian Pac J Cancer Prev. 2010;11(6):1631-6.

37. Wang JM, Xu B, Rao JY, Shen HB, Xue HC, Jiang QW. Diet habits, alcohol drinking, tobacco smoking, green tea drinking, and the risk of esophageal squamous cell carcinoma in the Chinese population. Eur J Gastroenterol Hepatol. 2007;19(2):171-6.

38. Yang CX, Wang HY, Wang ZM, Du HZ, Tao DM, Mu XY, et al. Risk factors for esophageal cancer: a case-control study in South-western China. Asian Pac J Cancer Prev. 2005:6(1):48-53.

39. De Stefani E, Boffetta P, Deneo-Pellegrini H, Ronco AL, Correa P, Mendilaharsu $\mathrm{M}$. The role of vegetable and fruit consumption in the aetiology of squamous cell carcinoma of the oesophagus: a case-control study in Uruguay. Int J Cancer. 2005;116(1):130-5.

40. De Stefani E, Boffetta P, Ronco AL, Deneo-Pellegrini H, Correa P, Acosta G, et al. Exploratory factor analysis of squamous cell carcinoma of the esophagus in Uruguay. Nutr Cancer. 2008;60(2):188-95.

41. De Stefani E, Deneo-Pellegrini H, Ronco AL, Boffetta P, Brennan P, Munoz N, et al. Food groups and risk of squamous cell carcinoma of the oesophagus: a case-control study in Uruguay. Br J Cancer. 2003;89(7):1209-14.

42. Sewram V, De Stefani E, Brennan P, Boffetta P. Mate consumption and the risk of squamous cell esophageal cancer in uruguay. Cancer Epidemiol Biomarkers Prev. 2003;12(6):508-13.

43. Castelletto R, Castellsague X, Munoz N, Iscovich J, Chopita N, Jmelnitsky A. Alcohol, tobacco, diet, mate drinking, and esophageal cancer in Argentina. Cancer Epidemiol Biomarkers Prev. 1994;3(7):557-64.

44. Szymanska K, Matos E, Hung RJ, Wunsch-Filho V, Eluf-Neto J, Menezes A, et al. Drinking of mate and the risk of cancers of the upper aerodigestive tract in Latin America: a case-control study. Cancer Causes Control. 2010;21(11):1799-806.

45. Rolon PA, Castellsague X, Benz M, Munoz N. Hot and cold mate drinking and esophageal cancer in Paraguay. Cancer Epidemiol Biomarkers Prev. 1995:4(6):595-605.

46. Khan NA, Teli MA, Mohib-UI Haq M, Bhat GM, Lone MM, Afroz F. A survey of risk factors in carcinoma esophagus in the valley of Kashmir, Northern India. J Cancer Res Ther. 2011;7(1):15-8.

47. Phukan RK, Chetia CK, Ali MS, Mahanta J. Role of dietary habits in the development of esophageal cancer in Assam, the north-eastern region of India. Nutr Cancer. 2001;39(2):204-9.

48. Srivastava M, Kapil U, Chattopadhyay TK, Shukla NK, Sundaram KR, Sekaran $\mathrm{G}$, et al. Nutritional factors in carcinoma oesophagus: a case-control study. Asia Pac J Clin Nutr. 1997;6(2):96-8.

49. Cook-Mozaffari PJ, Azordegan F, Day NE, Ressicaud A, Sabai C, Aramesh B. Oesophageal cancer studies in the Caspian Littoral of Iran: results of a case-control study. Br J Cancer. 1979;39(3):293-309.

50. Jessri M, Rashidkhani B, Hajizadeh B, Gotay C. Macronutrients, vitamins and minerals intake and risk of esophageal squamous cell carcinoma: a casecontrol study in Iran. Nutr J. 2011;10:137.

51. Cheng KK, Sharp L, McKinney PA, Logan RF, Chilvers CE, Cook-Mozaffari P, et al. A case-control study of oesophageal adenocarcinoma in women: a preventable disease. Br J Cancer. 2000;83(1):127-32.

52. Patel K, Wakhisi J, Mining S, Mwangi A, Patel R. Esophageal cancer, the topmost cancer at MTRH in the Rift Valley, Kenya, and its potential risk factors. ISRN Oncol. 2013;2013:503249.

53. Hanaoka T, Tsugane S, Ando N, Ishida K, Kakegawa T, Isono K, et al. Alcoho consumption and risk of esophageal cancer in Japan: a case-control study in seven hospitals. Jpn J Clin Oncol. 1994;24(5):241-6.

54. Freedman ND, Murray LJ, Kamangar F, Abnet CC, Cook MB, Nyren O, et al. Alcohol intake and risk of oesophageal adenocarcinoma: a pooled analysis from the BEACON Consortium. Gut. 2011;60(8):1029-37.

55. Lindkvist $B$, Johansen $D$, Stocks $T$, Concin $H$, Bjorge $T$, Almquist $M$, et al. Metabolic risk factors for esophageal squamous cell carcinoma and adenocarcinoma: a prospective study of 580,000 subjects within the Me-Can project. BMC Cancer. 2014;14:103.

56. Holmes RS, Vaughan TL. Epidemiology and pathogenesis of esophageal cancer. Semin Radiat Oncol. 2007;17(1):2-9.

57. Chen $X$, Yang CS. Esophageal adenocarcinoma: a review and perspectives on the mechanism of carcinogenesis and chemoprevention. Carcinogenesis. 2001;22(8):1119-29.

58. Mirvish SS. Role of N-nitroso compounds (NOC) and N-nitrosation in etiology of gastric, esophageal, nasopharyngeal and bladder cancer and contribution to cancer of known exposures to NOC. Cancer Lett. 1995;93(1):17-48. 
59. Breton JSF, Abbas A, Marnay J, Arsene D, Lechevrel M. Simultaneous use of DGGE and DHPLC to screen TP53 mutations in cancers of the esophagus and cardia from a European high incidence area (Lower Normandy, France). Mutagenesis. 2003;18:299-306.

60. Firouzeh Biramijamal AA, Parvin M, Hermann-Josef G, Reet K, Monica H. Unusual profile and high prevalence of p53 mutations in esophageal squamous cell carcinomas from Northern Iran. Cancer Res. 2001;61:3119-23.

61. Putz A, Hartmann AA, Fontes PR, Alexandre CO, Silveira DA, Klug SJ, et al. TP53 mutation pattern of esophageal squamous cell carcinomas in a high risk area (Southern Brazil): role of life style factors. Int J Cancer. 2002;98(1):99-105.

62. Sepehr A, Taniere P, Martel-Planche G, Zia'ee AA, Rastgar-Jazii F, Yazdanbod $M$, et al. Distinct pattern of TP53 mutations in squamous cell carcinoma of the esophagus in Iran. Oncogene. 2001;20(50):7368-74.

63. Wang LD, Zhou Q, Feng CW, Liu B, Qi YJ, Zhang YR, et al. Intervention and follow-up on human esophageal precancerous lesions in Henan, northern China, a high-incidence area for esophageal cancer. Gan To Kagaku Ryoho. 2002;29 Suppl 1:159-72.

64. Loria D, Barrios E, Zanetti R. Cancer and yerba mate consumption: a review of possible associations. Rev Panam Salud Publica. 2009;25(6):530-9.

65. Munoz N, Crespi M, Grassi A, Qing WG, Qiong S, Cai LZ. Precursor lesions of oesophageal cancer in high-risk populations in Iran and China. Lancet. 1982;1(8277):876-9.

66. Munoz N, Victora CG, Crespi M, Saul C, Braga NM, Correa P. Hot mate drinking and precancerous lesions of the oesophagus: an endoscopic survey in southern Brazil. Int J Cancer. 1987;39(6):708-9.

67. Wahrendorf J, Chang-Claude J, Liang QS, Rei YG, Munoz N, Crespi M, et al. Precursor lesions of oesophageal cancer in young people in a high-risk population in China. Lancet. 1989;2(8674):1239-41.

68. Ghadirian P. Thermal irritation and esophageal cancer in northern Iran. Cancer. 1987;60(8):1909-14.

69. Kuriyama S, Shimazu T, Ohmori K, Kikuchi N, Nakaya N, Nishino Y, et al. Green tea consumption and mortality due to cardiovascular disease, cancer, and all causes in Japan: the Ohsaki study. JAMA. 2006;296(10):1255-65.

70. Organization FaA: food balance sheets. http://www.fao.org/

71. Cheng KK, Day NE. Nutrition and esophageal cancer. Cancer Causes Control. 1996;7(1):33-40.

\section{Submit your next manuscript to BioMed Central and take full advantage of:}

- Convenient online submission

- Thorough peer review

- No space constraints or color figure charges

- Immediate publication on acceptance

- Inclusion in PubMed, CAS, Scopus and Google Scholar

- Research which is freely available for redistribution 